\title{
The influence of motor expertise on the brain activity of motor task performance: A meta-analysis of functional magnetic resonance imaging studies
}

\author{
Jie Yang
}

Published online: 2 December 2014

(C) Psychonomic Society, Inc. 2014

\begin{abstract}
Previous research has investigated the influence of long-term motor training on the brain activity of motor processes, but the findings are inconsistent. To clarify how acquiring motor expertise induces cortical reorganization during motor task performance, the current study conducted a quantitative meta-analysis on 26 functional magnetic resonance imaging (fMRI) studies that investigate motor task performance in people with long-term motor training experience (e.g., athletes, musicians, and dancers) and control participants. Meta-analysis of the brain activation in motor experts and novices showed similar effects in the bilateral frontal and parietal regions. The meta-analysis on the contrast between motor experts and novices indicated that experts showed stronger effects in the left inferior parietal lobule (BA 40) than did novices in motor execution and prediction tasks. In motor observation tasks, experts showed stronger effects in the left inferior frontal gyrus (BA 9) and left precentral gyrus (BA 6) than novices. On the contrary, novices had stronger effects in the right motor areas and basal ganglia as compared with motor experts. These results indicate that motor experts have effect increases in brain areas involved in action planning and action comprehension, and suggest that intensive motor training might elaborate the motor representation related to the task performance.
\end{abstract}

Keywords Practice-induced cortical reorganization - Motor expertise $\cdot$ Activation likelihood estimation · Meta-analysis . fMRI

\footnotetext{
J. Yang $(\triangle)$

ARC Center of Excellence in Cognition and its Disorders, Department of Cognitive Science, Macquarie University, Australian Hearing Hub, 16 University Avenue, North Ryde, NSW 2109, Australia

e-mail: j.yang@mq.edu.au
}

\section{Introduction}

People learn various new skills to meet challenges in work and daily life. To achieve learning goals, they need extensive training or practice. Practice enables the brain to fulfill a complex set of new requirements, such as sensorimotor processes, memory formation, and decision making.

Numerous studies have shown that the neural representation of adult cerebral cortex can change substantially as a result of practice (for reviews, see Bezzola, Mérillat, \& Jäncke, 2012; Herholz \& Zatorre, 2012; Jäncke, 2009a, b; Nakata, Yoshie, Miura \& Kudo, 2010; Zatorre, Fields, \& Johansen-Berg, 2012). Practice may induce an increase or a decrease of the brain activation associated with task performance, or it may result in a combination of activation increases and decreases across many brain areas, i.e., a functional reorganization of brain activity (Kelly \& Garavan, 2005). Practice-induced activity increases include the expansion of brain activity or increases in the strength of activation. These increases in activity have been found in task-specific areas such as primary and secondary sensory or motor cortex (e.g., Baeck et al., 2012), posterior parietal cortex related to motor planning (e.g., Calvo-Merino, Glaser, Grèzes, Passingham, \& Haggard, 2005; Kim et al., 2011; Stout, Passingham, Frith, Apel, \& Chaminade, 2011), and lateral temporal cortex associated with the storage of those representations (e.g., Calvo-Merino et al., 2005). Practice-induced activity decreases, on the other hand, could happen in the brain areas related to cognitive control or attentional processes, such as the prefrontal cortex (PFC) (Petrini et al., 2011) and anterior cingulate cortex (ACC) (e.g., Seo et al., 2012). These decreases in activity may reflect a more efficient use of specific neural circuits or the automation of task performance (Garavan, Kelley, Rosen, Rao, \& Stein, 2000). To summarize, practice-induced cortical reorganization is supposed to reflect the development of a new strategy to fulfill the task demand (e.g., Jonides, 2004; Kelly \& Garavan, 2005). 
One important approach to study practice-induced cortical reorganization is to recruit a professional population who have intensive motor training experience (e.g., athletes, pianists, and dancers) as participants and compare their brain activity during task performance with that of a control group (e.g., novices). Individuals who have achieved very high levels of motor skills and performance through long-term training provide such an opportunity.

Recent research has indicated that motor expertise influences the brain activity of motor execution (e.g., Bernardi et al., 2013; Meister et al., 2005), motor observation (e.g., Kim et al., 2011; Olsson \& Lundström, 2013; Pilgramm, Lorey, Stark, Munzert, Vaitl, \& Zentgraf, 2010; Stout et al., 2011; Wright, Bishop, Jackson, \& Abernethy, 2013), motor imagery or planning (e.g., Chang et al., 2011; Milton, Solodkin, Hlustík, \& Small, 2007; Wei \& Luo, 2010), motor prediction (e.g., Abreu et al., 2012; Balser et al., 2014; Bishop, Wright, Jackson, \& Abernethy, 2013), and even the comprehension of action language (Beilock, Lyons, MattarellaMicke, Nusbaum, \& Small, 2008; Lyons et al., 2010; Tomasino, Maieron, Guatto, Fabbro, \& Rumiati, 2013). However, the findings are inconsistent.

Several recent studies have found that compared with novices, motor experts showed reduced brain activity in the ventral and dorsal premotor cortex, supplementary motor area (SMA), superior parietal lobule (SPL), anterior cingulate cortex, basal ganglia, and cerebellum during motor tasks, and this may suggest enhanced brain functions in motor performance (e.g., Balser et al., 2014; Chang et al., 2011; Haslinger et al., 2004; Milton et al., 2007; Pau, Jahn, Sakreida, Domin, \& Lotze, 2013; Petrini et al., 2011; Wright, Bishop, Jackson, \& Abernethy, 2010). For instance, Milton et al. (2007) investigated the motor planning of expert golfers during pre-shot routine. The results showed that compared with novice golfers, expert golfers had stronger activation in the left dorsal premotor cortex, occipital cortex, and superior parietal lobule (SPL). However, novices showed a much more extended network than did experts, including anterior medial prefrontal cortex (amPFC), middle cingulate cortex (MCC), posterior cingulate cortex (PCC), basal ganglia, and anterior temporal lobe. The authors suggested that experts have developed a focused and efficient organization of task-related brain network through intensive training, whereas novices still need more resource to inhibit irrelevant information efficiently.

Petrini et al. (2011) investigated the brain activation of audio-visual processing (e.g., drumming) in drummers and novices. The results showed that when sound matched the observed movements, drummers indicated reduced activation in the bilateral middle frontal gyrus (MFG), right precentral gyrus, right inferior parietal lobule (IPL), right inferior temporal gyrus (ITG), left parahippocampal gyrus, and cerebellum. The two groups had similar brain activation when sound mismatched the observed movements. The authors suggested that perceptual and action expertise can result in the reduction of brain activation related to action-sound representations.

Haslinger et al. (2004) found that during performing mirror and parallel bimanual finger movements, professional pianists and naïve controls indicated different brain activation patterns. Direct comparison between the two groups in the parallel movement condition showed that musically naïve controls had stronger activation in the right MFG, right dorsal premotor cortex, right inferior temporal gyrus (ITG), ACC, and cerebellum than did pianists. During the performance of parallel compared to mirror movements, musically naïve controls showed stronger activity than did pianists in an extended network, including the left ventral premotor cortex, bilateral PFC, right SMA, right ITG, bilateral lingual gyrus, and cerebellum. The authors claim that pianists have developed more efficient cortical and subcortical systems for bimanual motor control as compared with novices.

On the other hand, several studies have shown that motor experts indicated activation increase in brain areas related to their expertise during task performance (Calvo-Merino et al., 2005; Kim et al., 2011; Pilgramm et al., 2010; Wei \& Luo, 2010; Wright et al., 2010). For example, Wei and Luo (2010) found that expert divers showed stronger activation in the left parahippocampal gyrus and right fusiform gyrus (FG) in diving imagery than in gymnastic imagery, and that experts showed stronger activation in the bilateral ITG in diving observation than in gymnastic imagery. No such effects were found in novices. These results may suggest that during motor imagery related to their expertise, motor experts use their kinesthetic imagery more efficiently than do novices.

Calvo-Merino et al. (2005) reported that during the observation of their dance style, expert dancers showed stronger activation in the bilateral premotor cortex, bilateral intraparietal sulcus (IPS), right SPL, and left posterior superior temporal sulcus (STS) as compared with watching the dance styles they had not been trained to perform. The authors suggest that an individual's personal motor repertoire is used to help understand observed actions.

Kim et al. (2011) found that during action observation, expert archers showed stronger activation in the bilateral premotor cortex, dorsal medial prefrontal cortex (dmPFC), inferior parietal cortex, STS, ACC, PCC, caudate, and parahippocampal gyrus than did novices. According to the authors, the stronger premotor effects indicated enhanced motor repertoire in the mirror system in experts, the increased prefrontal effects showed the engagement of theory of the mind in experts, and the stronger posterior cingulate effects indicated episodic recall from familiar and meaningful information during motor observation.

Taken together, the above evidence has shown that intensive motor training can change activity in task-related areas and areas related to attentional processes and episodic retrieval during task performance, but the activity pattern is still 
unclear. To clarify the practice-induced functional reorganization in the brain, the current study conducted a quantitative meta-analysis on the functional magnetic resonance imaging (fMRI) studies that investigate how motor expertise influences brain activity during task performance. We examined the general influence of motor expertise on motor task performance, and the effects of motor expertise in different motor tasks. The activation likelihood estimation (ALE; Eickhoff, Laird, Grefkes, Wang, Zilles, \& Fox, 2009) method was used to perform the meta-analysis. In the current study, we labeled people that have long-term motor training experience motor experts. The term was chosen according to previous research (e.g., motor-skill experts, Allard \& Starkes, 1991). In Allard and Starkes (1991), the term motor-skill experts refers to people with long-term motor training experience in sports, dance, and other domains. This is a general term and does not describe individual motor expertise. We used the term in this study for the sake of simpliness.

According to the view of practice-induced cortical reorganization, motor training experience may result in a more efficient use of task-specific areas and the automation of task performance (Baeck et al., 2012; Garavan et al., 2000). First, we predicted that compared with novices, motor experts might indicate an activity increase in the fronto-parietal areas, including the ventral premotor cortex involved in action observation (Rizzolatti, Cattaneo, Fabbri-Destro, \& Rozzi, 2014), posterior parietal cortex involved in motor planning (Grezes \& Decety, 2002; Konen et al., 2013), and M1 involved in action execution. Second, we predicted that motor experts might indicate an activity decrease in the areas associated with motor control, motor selection, and attentional processes (e.g., medial PFC, ACC, and basal ganglia). Third, we predicted that since motor experts have rich experience and memory of their training, they might show additional activity in the cortical areas involved in episodic memory retrieval, such as retrosplenial cortex and PCC during motor task performance as compared with novices (Kim et al., 2011). The opposite hypothesis is that no significant difference would be found between motor experts and novices.

\section{Material and methods}

\section{Paper selection}

We searched papers from the PubMed database (http:/www. ncbi.nlm.nih.gov/pubmed/) and ScienceDirect database (http://www.sciencedirect.com/). The keywords included three parts: the research field (e.g., "action expertise," "action skill," "motor expertise," "sport expertise," "sport skill," or "sport experience"); the investigation of brain functions (e.g., "brain" or "cortical"), and the neuroimaging technique (e.g., "fMRI," "functional MRI," or "functional magnetic resonance imaging"). We identified 39 studies investing brain activations in motor experts and novices, and then we selected studies for the current meta-analysis using the following criteria:

1. Studies using the fMRI technique and collecting data from healthy adults were selected. We excluded studies that use other techniques (PET, TMS, MEG, and EEG) and just focused on fMRI to make sure that all original studies in this meta-analysis have approximate spatial resolution.

2. Studies recruiting a group of motor experts (e.g., athletes, dancers, or pianists) and a group of novices were selected. This is because the aim of the current meta-analysis is to investigate the brain activity in both motor experts and novices. Furthermore, this ensures that for each task included in the meta-analysis, brain activations for both motor experts and novices are available, and hence enables the meta-analysis to show the clear influence of motor expertise on the brain activation of task performance. Additionally, selecting studies that recruit both motor experts and novices make the numbers of the two types of subjects close, and thus help reduce the influence of subject number on the final ALE effects. For those reasons, we excluded studies that only recruited an expert group or a non-expert group. If a study recruited a group of experts, a group of novices, and a group of training people whose motor skill level is between experts and novices, then only experts' and novices' brain activation results were used as the input of the meta-analysis.

3. Studies using motor-related tasks were selected. The tasks included motor execution tasks that are directly related to training experience, and other motor-related tasks, such as motor imagery, motor observation, motor planning, motor anticipation, and the comprehension of action language.

4. Studies reporting motor experts' and novices' brain activation results were selected. Studies that only reported the brain activation differences between motor experts and novices were also selected. In either case, the standard coordinates of activation foci must be reported in the Talairach space (Talairach \& Tournoux, 1988) or Montreal Neurologic Institute (MNI) space.

5. Studies reporting significant results in whole-brain analysis were selected. Studies that only reported significant effects in regions of interest (ROIs) were excluded.

Twenty-six studies met all the criteria and were included in the meta-analysis (Table 1).

\section{Selection of contrasts}

To explore the general influence of motor expertise on motor task performance, we selected four types of contrasts from the 
Table 1 Original fMRI studies included in the meta-analysis

\begin{tabular}{|c|c|c|c|c|c|c|c|c|c|c|c|}
\hline Authors & Year & $\begin{array}{l}\text { Field } \\
\text { strength }\end{array}$ & Voxel size $\left(\mathrm{mm}^{3}\right)$ & Category & Expertise & Task & $\begin{array}{l}\text { Expert } \\
(\mathrm{N})\end{array}$ & $\begin{array}{l}\text { Novice } \\
\text { (N) }\end{array}$ & $\begin{array}{l}\text { Foci- } \\
\text { expert }\end{array}$ & $\begin{array}{l}\text { Foci- } \\
\text { novice }\end{array}$ & $\begin{array}{l}\text { Foci- } \\
\text { contrast }\end{array}$ \\
\hline Abreu et al. & 2012 & $3 \mathrm{~T}$ & $3 \times 3 \times 3.8$ & sport & playing basketball & motor prediction & 16 & 16 & yes & yes & \\
\hline Balser et al. & 2014 & $1.5 \mathrm{~T}$ & Slice thickness $=5 \mathrm{~mm}$ & sport & playing tennis & motor prediction & 16 & 16 & yes & yes & yes \\
\hline Baumann et al. & 2007 & $3 \mathrm{~T}$ & $2.75 \times 2.75 \times 4.5$ & music & playing piano & motor execution & 7 & 7 & & & yes \\
\hline $\begin{array}{l}\text { Berkowitz and } \\
\text { Ansari }\end{array}$ & 2010 & $3 \mathrm{~T}$ & $4 \mathrm{~mm}$ thickness & music & playing piano & motor execution & 13 & 15 & & & yes \\
\hline Bernardi et al. & 2013 & $1.5 \mathrm{~T}$ & Slice thickness $=5 \mathrm{~mm}$ & sport & driving racing-car & motor execution & 11 & 11 & & & yes \\
\hline Bishop et al. & 2013 & $3 \mathrm{~T}$ & $3 \mathrm{~mm}$ thickness & sport & playing soccer & motor prediction & 14 & 11 & & & yes \\
\hline Chang et al. & 2011 & $3 \mathrm{~T}$ & $4 \mathrm{~mm}$ thickness & sport & archery & motor imagery & 18 & 18 & yes & yes & yes \\
\hline Harris and de Jong & 2014 & $3 \mathrm{~T}$ & $3.5 \times 3.5 \times 3.5$ & music & Playing keyboard & motor imagery & 12 & 12 & & & yes \\
\hline Hasegawa et al. & 2004 & $1.5 \mathrm{~T}$ & Pixel dimension $3.44 \times 3.44$ & music & playing piano & motor observation & 9 & 7 & yes & yes & \\
\hline Haslinger et al & 2004 & $1.5 \mathrm{~T}$ & Slice thickness $=5 \mathrm{~mm}$ & music & playing piano & motor execution & 12 & 12 & & & yes \\
\hline Haslinger et al & 2005 & $1.5 \mathrm{~T}$ & Slice thickness $=5 \mathrm{~mm}$ & music & playing piano & motor observation & 12 & 12 & & & yes \\
\hline Kim et al. & 2011 & $3 \mathrm{~T}$ & $4 \mathrm{~mm}$ thickness & sport & archery & motor observation & 20 & 21 & yes & yes & yes \\
\hline Kim et al. & 2008 & $3 \mathrm{~T}$ & $?$ & sport & archery & motor planning & 8 & 8 & yes & yes & \\
\hline $\begin{array}{l}\text { Landau and } \\
\text { D'esposito }\end{array}$ & 2006 & $4 \mathrm{~T}$ & $3.5 \mathrm{~mm}$ thickness & music & playing piano & motor execution & 9 & 8 & yes & yes & yes \\
\hline Lee and Noppeney & 2011 & $3 \mathrm{~T}$ & $3 \times 3 \times 3.3$ & music & playing piano & music listening & 18 & 19 & & & yes \\
\hline Meister et al. & 2005 & $1.5 \mathrm{~T}$ & Slice thickness $=5 \mathrm{~mm}$ & music & playing piano & motor execution & 12 & 12 & yes & yes & yes \\
\hline $\begin{array}{l}\text { Olsson and } \\
\text { Lundstrom }\end{array}$ & 2013 & $3 \mathrm{~T}$ & $3.4 \mathrm{~mm}$ thickness & sport & playing hockey & motor observation & 3 & 3 & yes & yes & \\
\hline Pau et al. & 2013 & $3 \mathrm{~T}$ & $3 \times 3 \times 3$ & music & playing piano & motor execution & 14 & 15 & & & yes \\
\hline Petrini et al. & 2011 & $3 \mathrm{~T}$ & $4 \mathrm{~mm}$ thickness & music & drumming & motor prediction & 11 & 11 & & & yes \\
\hline Pilgramm & 2010 & $1.5 \mathrm{~T}$ & $?$ & sport & dancing & motor observation & 18 & 18 & & & yes \\
\hline Seo et al. & 2012 & $3 \mathrm{~T}$ & $4 \mathrm{~mm}$ thickness & sport & archery & visuo-spatial memory & 20 & 23 & yes & yes & yes \\
\hline Stout et al. & 2011 & $3 \mathrm{~T}$ & $2 \mathrm{~mm}$ thickness & other & making tools & motor observation & 5 & 11 & yes & yes & \\
\hline Tomasino et al. & 2013 & $3 \mathrm{~T}$ & $4 \mathrm{~mm}$ thickness & sport & playing volleyball & motor language processing & 10 & 10 & & & yes \\
\hline Wei and Luo & 2010 & $3 \mathrm{~T}$ & $4 \mathrm{~mm}$ thickness & sport & diving & motor imagery & 12 & 12 & yes & yes & \\
\hline Wright et al. & 2013 & $3 \mathrm{~T}$ & $3 \mathrm{~mm}$ thickness & sport & playing soccer & motor observation & 17 & 17 & yes & yes & yes \\
\hline Wright et al. & 2011 & $3 \mathrm{~T}$ & $3 \mathrm{~mm}$ thickness & sport & playing badminton & motor prediction & 8 & 8 & & & yes \\
\hline
\end{tabular}

26 studies. The first type of contrast showed that motor experts indicated stronger activation in motor tasks than in non-motor baseline tasks. The second type of contrast showed that novices had stronger activation in motor tasks than in non-motor baseline tasks. The third type of contrast showed that motor experts indicated stronger activation than did novices during motor task performance. The fourth type of contrast showed that novices had stronger activation than did motor experts during motor task performance.

To examine the effects of motor expertise in different motor task groups, we divided the original studies into three motor task groups, including motor execution, motor observation, and motor prediction. We focused on the contrast between motor experts and novices within each task group because it was the main research interest of the current study. The meta-analyses in each task group included an analysis for the contrast showing stronger effects in motor experts than in novices, and an analysis for the contrast showing stronger effects in novices than in motor experts. Because meta-analysis cannot be conducted on a single study, motor imagery studies were not grouped together as a motor task group (only one study shows stronger effects in motor experts than in novices, Harris \& de Jong, 2014; and only one study shows stronger effects in novices than in motor experts, Chang et al., 2011).

ALE meta-analyses ALE meta-analysis was performed with Ginger ALE 2.3.1 software (http://www.brainmap.org/ ale/) (Eickhoff et al., 2009) and conducted in the standard Talairach space. If the coordinates of foci were reported in the MNI space, we then transformed them into the standard Talairach space using the icbm2tal transformation (Lancaster et al., 2007). In the ALE meta-analysis for each condition group, the selected foci and subject number of each original study were used as input.

As an important algorithm for coordinate-based meta-analysis, ALE considers each included activation focus as a center of probability distribution, rather than a point in the voxelscaled brain. It assumes that the brain activation is most likely located in the reported coordinate, but the activation might also be in the vicinity of the coordinate. In this way, each reported coordinate indicated activation information in a dimensional way (i.e., with some possibilities), but not in a binary way (i.e., the activation is here or not). 
First, ALE modeled the included foci as centers of 3D Gaussian probability distribution that could capture the spatial uncertainty associated with each focus (Eickhoff et al., 2009). Two types of spatial uncertainty were acknowledged, including uncertainty caused by between-subject variances, and uncertainty caused by between-laboratory variances. Furthermore, the width of the spatial uncertainty of each focus was adjusted by the number of participants in each study: the more participants in a study, the bigger the likelihood of true activation located in the foci reported in the study. Foci from studies with a large number of participants have a smaller spatial uncertainty and a larger influence on the results of the meta-analysis than do foci from studies with small number of participants.

Second, the probability distributions of all included foci in one study were combined for each voxel and a modeled activation map was generated. The final ALE map was generated from the activation maps of all included studies, and it contained an ALE score for each voxel. The ALE score values were assessed for statistical significance. A random-effects analysis (i.e., testing for above-chance clustering of experiments, rather than foci) was conducted to identify the significant ALE effect (Eickhoff et al., 2009), and an image that contains the $p$ value of each voxel was generated. The random-effects analysis allows conclusions beyond the studies included in the meta-analysis (Penny \& Holmes, 2004).

Third, the computed ALE map was compared with a null distribution to distinguish random convergence and true convergence. The null distribution reflects a random spatial association between the activation maps (i.e., the included experiments converged entirely by chance). Voxels in which activation maps converged more robustly than expected in the null distribution were identified. A cluster analysis was conducted at the threshold of $p<0.05$ (False Discovery Rate correction) with a default cluster size in $200 \mathrm{~mm}^{3}$.

\section{Results}

General effects for motor expertise

Motor experts: motor task performance $>$ baseline task performance The meta-analysis in this condition included 13 studies with 256 foci. The results showed 13 significant clusters. These clusters were located in the left precentral gyrus (BA 4/6), bilateral inferior frontal gyrus (IFG) (BA 9), bilateral MFG (BA 6), left superior frontal gyrus (SFG) (BA 6), bilateral IPL/superior parietal lobule (SPL) (BA 40/7), bilateral precuneus (BA 7), right cingulate gyrus (BA 32), and claustrum (Table 2, Fig. 1)
Novices: motor task performance > baseline task performance The meta-analysis in this condition included 13 studies with 250 foci. The result showed ten significant clusters in the novice group. The effects were located in the right precentral gyrus (BA 6), bilateral medial frontal gyrus (BA 6/32), bilateral MFG (BA 6), bilateral IFG (BA 9), bilateral SPL (BA 7), bilateral precuneus (BA 7), bilateral IPL (BA 40), left supramarginal gyrus (SMG) (BA 40), and cerebellum (Table 3 and Fig. 2).

Contrast: motor experts $>$ novices The meta-analysis in this condition included 15 studies with 251 foci. The results indicated 16 significant clusters in this contrast group, and they were located in the left IFG (BA 9), bilateral precentral gyrus (BA 6), left MFG (BA 6), left SFG (BA 6), bilateral SPL (BA 7), left IPL (BA 40), right precuneus (BA 7), left superior temporal gyrus (STG) (BA 38/22/42/13), and cerebellum (Table 4 and Fig. 3).

Contrast: novices $>$ motor experts The meta-analysis in this condition included six studies with 78 foci. The meta-analysis showed four significant clusters in this contrast group, and they were located in the right precentral gyrus (BA 6), right lingual gyrus (BA 18), right lentiform nucleus, and cerebellum (Table 4 and Fig. 4).

Effects of motor expertise in different motor task groups

Motor execution The meta-analysis within the motor execution task group showed that motor experts had stronger effects in the left IPL (BA 40) than novices. On the contrary, novices had stronger effects in the left cerebellum and right putamen.

Motor observation Within the motor observation task group, the meta-analysis showed that motor experts had stronger effects in the left precentral gyrus (BA 6) and left IFG (BA 9 ) as compared with novices. No stronger effect in novices was found.

Motor prediction Within the motor prediction task group, the meta-analysis showed that motor experts had a stronger effect in the left IPL (BA 40). On the other hand, novices had a stronger effect in the left precuneus (BA 7).

Table 5 shows the effects of motor expertise in different motor tasks.

\section{Discussion}

Previous studies have found that intensive motor training can induce functional reorganization in the brain during motor task performance (Bernardi et al., 2013; Kim et al., 2011; 
Table 2 Meta-analysis results from the expert group

\begin{tabular}{|c|c|c|c|c|c|c|}
\hline Volume $\left(\mathrm{mm}^{3}\right)$ & $\mathrm{x}$ & $\mathrm{y}$ & $\mathrm{z}$ & Hemisphere & Region & BA \\
\hline \multirow[t]{6}{*}{4552} & -36 & -46 & 38 & Left & Inferior Parietal Lobule & 40 \\
\hline & -24 & -54 & 50 & Left & Precuneus & 7 \\
\hline & -30 & -56 & 42 & Left & Inferior Parietal Lobule & 7 \\
\hline & -24 & -58 & 50 & Left & Precuneus & 7 \\
\hline & -28 & -54 & 50 & Left & Precuneus & 7 \\
\hline & -32 & -48 & 52 & Left & Superior Parietal Lobule & 7 \\
\hline \multirow[t]{2}{*}{3216} & 40 & -40 & 54 & Right & Inferior Parietal Lobule & 40 \\
\hline & 34 & -50 & 44 & Right & Inferior Parietal Lobule & 40 \\
\hline \multirow[t]{2}{*}{2312} & -22 & -12 & 54 & Left & Middle Frontal Gyrus & 6 \\
\hline & -36 & -16 & 62 & Left & Precentral Gyrus & 4 \\
\hline \multirow[t]{2}{*}{2192} & 26 & -12 & 54 & Right & Middle Frontal Gyrus & 6 \\
\hline & 24 & -6 & 58 & Right & Middle Frontal Gyrus & 6 \\
\hline 1072 & -52 & 6 & 26 & Left & Inferior Frontal Gyrus & 9 \\
\hline 1000 & 54 & 8 & 32 & Right & Inferior Frontal Gyrus & 9 \\
\hline \multirow[t]{2}{*}{552} & 0 & 12 & 48 & Left & Superior Frontal Gyrus & 6 \\
\hline & 6 & 8 & 42 & Right & Cingulate Gyrus & 32 \\
\hline 488 & 22 & -66 & 50 & Right & Precuneus & 7 \\
\hline 480 & -38 & -6 & 36 & Left & Precentral Gyrus & 6 \\
\hline 472 & 4 & -10 & 64 & Right & Medial Frontal Gyrus & 6 \\
\hline 248 & -32 & 16 & 4 & Left & Claustrum & \\
\hline 248 & 12 & -24 & 14 & Right & Thalamus & \\
\hline 200 & 30 & 18 & 6 & Right & Claustrum & \\
\hline
\end{tabular}

Note: The coordinates of the effects are in the Talairach space

Meister et al., 2005; Olsson \& Lundström, 2013; Pilgramm et al., 2010; Stout et al., 2011; Wright et al., 2013). Compared with novices, motor experts showed activity change in motor areas (e.g., Baeck et al., 2012), areas related to attentional processes (Petrini et al., 2011; Seo et al., 2012), areas involved in episodic retrieval during motor task performance (Kim et al., 2011), and subcortical areas related to motor control, such as putamen (Baeck et al., 2012; Landau \& D'esposito, 2006), caudate (Landau \& D'esposito, 2006; Kim et al., 2011; Wright et al., 2011), and thalamus (Bishop et al., 2013; Landau \& D'esposito, 2006). Although these findings have confirmed the influence of motor experience on the brain activity of motor task performance, the pattern of functional reorganization (i.e., the combination of activity increase and decrease) is still unclear. Activity increases may reflect the recruitment of additional cortical areas during task performance, and activity decreases may indicate a more efficient use of specific neural circuits related to practice (for a review, see Kelly \& Garavan, 2005). Clarifying the way that motor expertise changes activity in these areas can help understand practiceinduced cortical reorganization.

To achieve this goal, the current study conducted a quantitative meta-analysis on the fMRI studies that investigate how people with long-term motor training experience and novices perform motor tasks. To explore the general influence of motor expertise on the brain activity of motor task performance, we computed the ALE effects for motor experts' brain response, novices' brain response, and the effects for the contrast between motor experts and novices. To examine the effects of motor expertise in different motor task groups, we computed the ALE effect for the contrast between motor experts and novices in the motor execution task group, motor observation task group, and motor prediction task group.

The effect of general motor expertise and the effect of motor expertise in motor observation both indicate that motor experts have stronger effects in the left IFG/precentral gyrus (BA 9/6). This confirms previous results that motor experts show stronger activation in the premotor cortex as compared with novices (e.g., Baeck et al., 2012; Calvo-Merino et al., 2005; Pilgramm et al., 2010). The effects may suggest that intensive motor training strengthens the motor representation of a specific motor performance (Baeck et al., 2012). It is worth noting that in the current meta-analysis, motor experts indicated stronger effects in both ventral and dorsal premotor areas than did novices. Pilgramm et al. (2010) examined the influences of motor expertise and visual viewpoint (e.g., internal vs. external viewpoint) on the activity of the ventral and dorsal premotor cortex during motor observation. The authors 

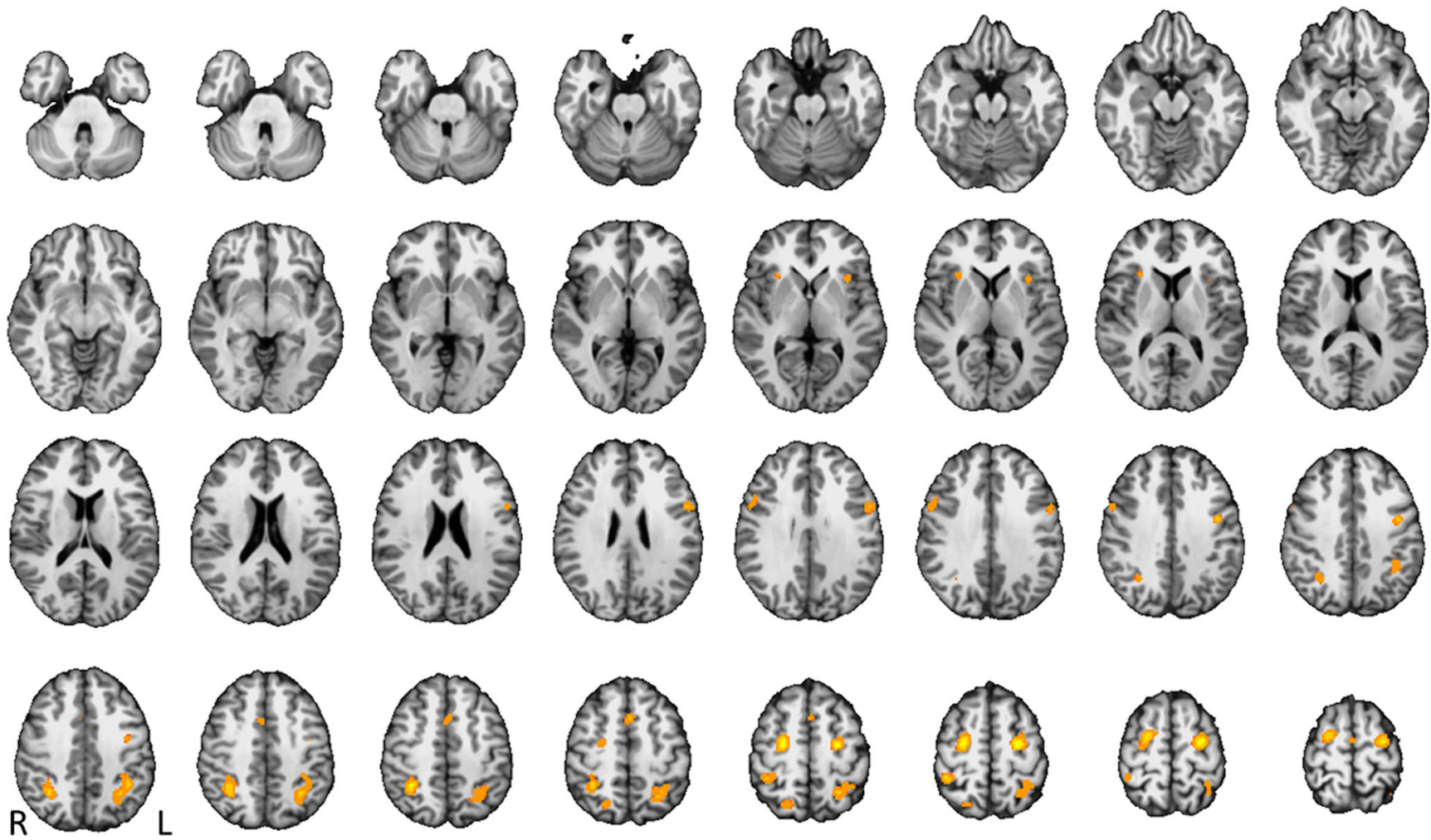

Fig. 1 Activation likelihood estimation (ALE) effects in the motor expert group. $L=$ left, $R=$ right

found a functional dissociation between the ventral and dorsal premotor cortex: motor experts revealed increased activity in the ventral premotor cortex, whereas visual viewpoint influenced activity in both ventral and dorsal premotor cortex. Previous studies have shown that the premotor cortex plays a key role in the sensory guidance of motor behavior (e.g., Chouinard \& Paus, 2006). The ventral premotor cortex is important for matching an observed motor act (i.e., sensory information) with an executed action (Hoshi \& Tanji, 2007; Newman-Norlund, van Schie, van Zuijlen, \& Bekkering, 2007).

The stronger effects in IFG (BA 9) and left precentral gyrus (BA 6) in the motor observation group suggest that as a part of the mirror neuron system (for a review, see Rizzolatti et al., 2014), these areas are more involved in understanding others' action goals after long-term motor training. For instance, intensive motor training may develop specific internal sensorimotor representation, which can make the matching between sensory information and one's own motor repertoire very elaborate. This elaborate matching process may increase activity in the ventral premotor cortex (Pilgramm et al., 2010). The dorsal premotor cortex has been found to be involved in motor control (Hoshi \& Tanji, 2007). Motor experts may have developed more complex motor control during motor task performance as compared with novices, and this may strengthen the activity in the dorsal premotor cortex. Although the current meta-analysis shows that motor expertise influences effects in the dorsal and ventral premotor cortex, it does not allow us to investigate the functional dissociation between the two areas, and thus future work is require to address the issue.

Compared with novices, motor experts also indicate stronger effects in the posterior parietal cortex (PPC) in motor execution and motor prediction task groups. This area plays an important role in producing planned movements, such as reaching, grasping, and tool use (for a review, see Vingerhoets, 2014). Previous studies have indicated that PPC receives input from the auditory cortex, visual cortex, and somatosensory cortex to localize the body and external objects in space during motor planning (e.g., Binkofski et al., 1999; Decety et al., 1994; Grafton, Arbib, Fadiga, \& Rizzolatti, 1996; Grezes \& Decety, 2002; Konen et al., 2013), and that the output of PPC goes to the dorsal lateral PFC and premotor cortex (e.g., Johnson, Ferraina, Bianchi, \& Caminiti, 1996). Because this area plays a key role in motor planning, one possibility is that intensive motor training may develop efficient motor planning in task performance and thus increases activity in the PPC (e.g., Calvo-Merino et al., 2005; Kim et al., 2011; Stout et al., 2011). On the other hand, motor training might induce the automation of task performance and simplify the motor planning process, thereby decreasing activity in the PPC (e.g., Petrini et al., 2011). In the current study, motor experts showed a stronger PPC effect as compared with novices when performing trained actions (Bernardi et al., 2013; Pau et al., 2013), observing other people doing the 
Table 3 Meta-analysis results from the novice group

\begin{tabular}{llllll}
\hline Volume $\left(\mathrm{mm}^{3}\right)$ & $\mathrm{x}$ & $\mathrm{y}$ & Hemisphere & Region \\
\hline 5784 & -24 & -8 & 54 & Left & Middle Frontal Gyrus \\
& -6 & -2 & 58 & Left & Medial Frontal Gyrus \\
& 2 & -6 & 64 & Right & Medial Frontal Gyrus \\
& -2 & 4 & 46 & Left & Medial Frontal Gyrus \\
& -2 & 12 & 46 & Left & Medial Frontal Gyrus \\
4288 & -30 & -54 & 50 & Left & Superior Parietal Lobule \\
& -34 & -42 & 42 & Left & Inferior Parietal Lobule \\
& -26 & -56 & 54 & Left & Precuneus \\
3368 & 32 & -44 & 44 & Right & Inferior Parietal Lobule \\
& 30 & -52 & 44 & Right & Superior Parietal Lobule \\
2656 & 24 & -14 & 54 & Right & Middle Frontal Gyrus \\
872 & 10 & -72 & 44 & Right & Precuneus \\
848 & 40 & 0 & 32 & Right & Precentral Gyrus \\
736 & 50 & 4 & Right & Inferior Frontal Gyrus \\
336 & -12 & -68 & 50 & Left & Precuneus \\
240 & -50 & 4 & Left & Inferior Frontal Gyrus \\
232 & 30 & -52 & -24 & Right & Cerebellum \\
\hline
\end{tabular}

Note: The coordinates of the effects are in the Talairach space

trained actions (Haslinger et al., 2005), or predicting object movement on the basis of training experience (Balser et al., 2014). This is in line with the former view and implies that motor experts, after intensive training, may be better at integrating somatosensory, auditory, and visual information in location monitoring and motor adjustment (Baeck et al.,
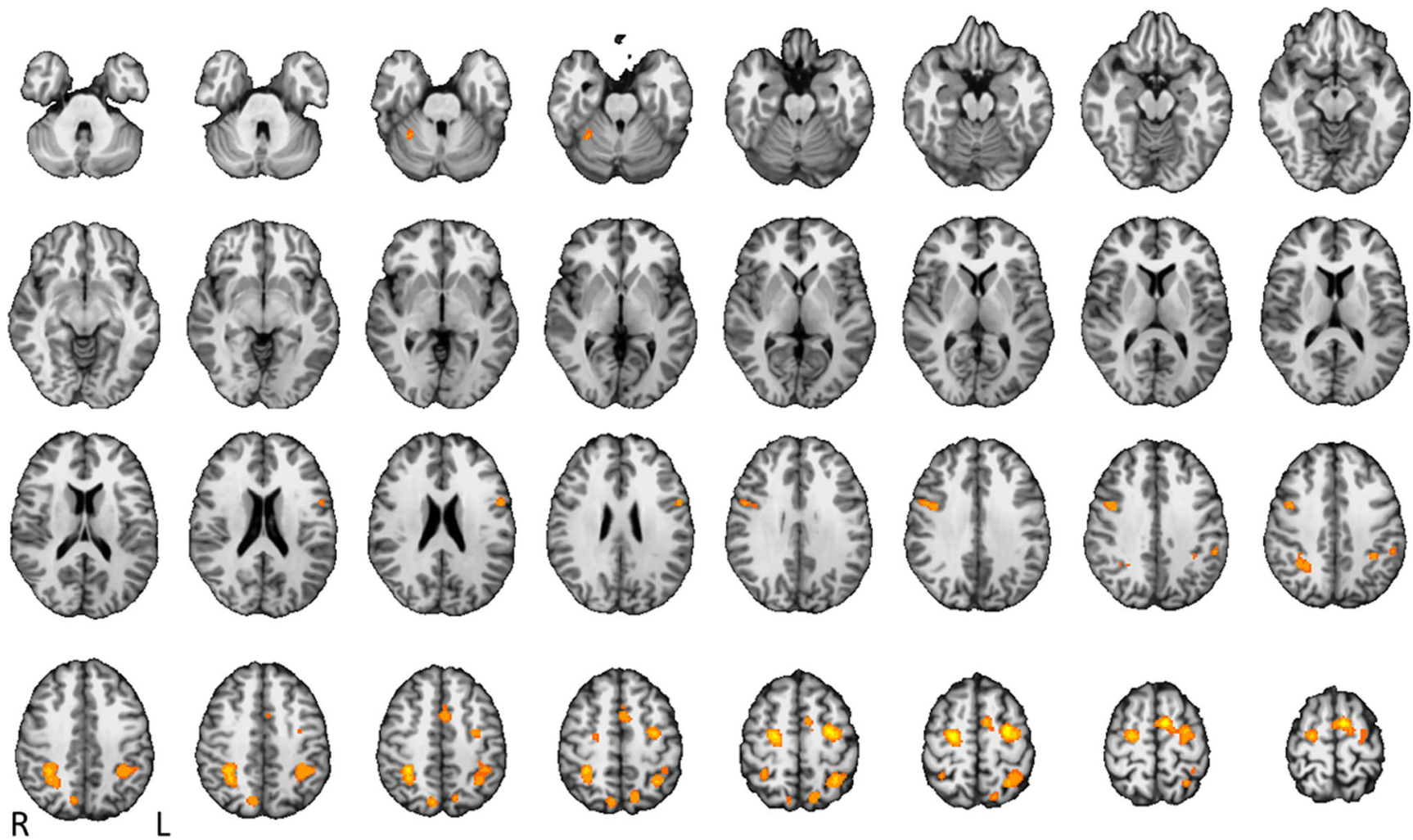

Fig. 2 Activation likelihood estimation (ALE) effects in the novice group. $L=$ left, $R=$ right 
Table 4 Meta-analysis results of the differences between motor experts and novices

\begin{tabular}{|c|c|c|c|c|c|c|}
\hline Volume $\left(\mathrm{mm}^{3}\right)$ & $\mathrm{x}$ & $\mathrm{y}$ & $\mathrm{z}$ & Hemisphere & Region & BA \\
\hline \multicolumn{7}{|c|}{ Motor experts $>$ novices } \\
\hline \multirow[t]{4}{*}{3344} & -50 & 0 & 38 & Left & Precentral Gyrus & 6 \\
\hline & -48 & 0 & 32 & Left & Precentral Gyrus & 6 \\
\hline & -56 & 6 & 32 & Left & Precentral Gyrus & 6 \\
\hline & -42 & 8 & 44 & Left & Middle Frontal Gyrus & 8 \\
\hline 656 & 0 & 4 & 58 & Left & Superior Frontal Gyrus & 6 \\
\hline 552 & 24 & -16 & 52 & Right & Precentral Gyrus & 6 \\
\hline 544 & -30 & -54 & 54 & Left & Superior Parietal Lobule & 7 \\
\hline 536 & 32 & -48 & 50 & Right & Superior Parietal Lobule & 7 \\
\hline 448 & 48 & 10 & 30 & Right & Inferior Frontal Gyrus & 9 \\
\hline 408 & -44 & -34 & 44 & Left & Inferior Parietal Lobule & 40 \\
\hline 328 & -34 & -46 & 38 & Left & Inferior Parietal Lobule & 40 \\
\hline 320 & 26 & -10 & 64 & Right & Precentral Gyrus & 6 \\
\hline 296 & 12 & -68 & 42 & Right & Precuneus & 7 \\
\hline 280 & 10 & -74 & 38 & Right & Precuneus & 7 \\
\hline \multirow[t]{2}{*}{264} & -52 & 6 & -8 & Left & Superior Temporal Gyrus & 38 \\
\hline & -50 & 10 & -6 & Left & Superior Temporal Gyrus & 22 \\
\hline 264 & -8 & -12 & 56 & Left & Medial Frontal Gyrus & 6 \\
\hline 232 & -58 & -30 & 14 & Left & Superior Temporal Gyrus & 42 \\
\hline \multicolumn{7}{|c|}{ Novices $>$ motor experts } \\
\hline 336 & -4 & -80 & -12 & Left & Cerebellum & \\
\hline 312 & 52 & -4 & 38 & Right & Precentral Gyrus & 6 \\
\hline 224 & 6 & -84 & -4 & Right & Lingual Gyrus & 18 \\
\hline \multirow[t]{2}{*}{208} & 24 & -2 & 14 & Right & Lentiform Nucleus & \\
\hline & 22 & 2 & 16 & Right & Lentiform Nucleus & \\
\hline
\end{tabular}

Note: The coordinates of the effects are in the Talairach space

2012). Thus, motor experts may have developed a more complex motor planning process that can serve various tasks related to their motor experience.

Compared with novices, motor experts indicated effect decreases in several brain regions, including the right precentral gyrus (BA 6), right lingual gyrus (BA 18), and basal ganglia. These decreased effects are consistent with previous research (e.g., Milton et al., 2007; Haslinger et al., 2004), and might suggest that motor experts have developed more efficient cortical and subcortical systems during motor training. However, it is hard to conclude that motor experts use less cognitive control or attentional processes when performing motor tasks, because the motor expert group showed no decreases in PFC and ACC that are involved in cognitive control functions. One possible explanation for the lack of effect decreases in the PFC or ACC is that the motor tasks used in these studies are not entirely the same as those practiced by motor experts. The motor observation, motor imagery, and motor prediction tasks can be strongly related to the motor training (e.g., participants were asked to observe, imagine, or anticipate the trained sport actions), and thus significant enhanced effects in motor areas are found in motor experts as compared with novices. However, motor experts might have not been trained on the exact tasks that were used in fMRI studies, and thus they might not be able to perform these tasks automatically. In addition, the experimental settings in these fMRI studies could be very different from the real sport game settings, and motor experts might still need cognitive control or attention processes to finish the motor tasks. In other words, the involvement of cognitive control processes could be influenced by the similarities between the motor tasks and motor training.

These contrasting results showed that motor experts and novices had different effects in the cerebellum. The cerebellum has been found to be involved in motor learning and temporal processing of movements (Llinás, 2011; Stoodley, 2012). Previous research has indicated that motor training can modulate cerebellar activation. For instance, Koeneke et al. (2004) found that while performing unimanual and bimanual finger movements, skilled keyboard players showed less cerebellar activity than did control participants. The authors conclude that motor training reduces the number of neurons 

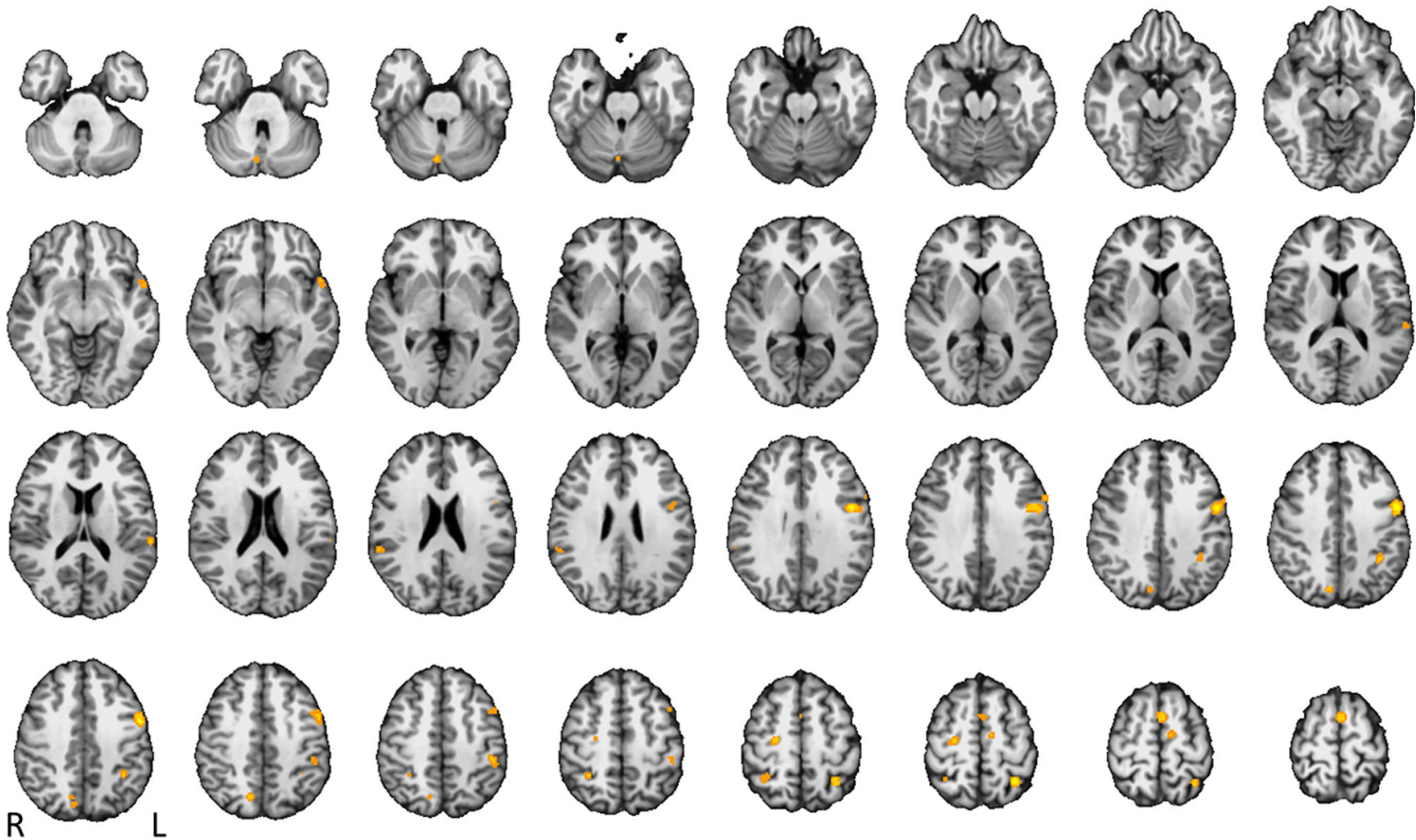

Fig. 3 Stronger activtion likelihood estimation (ALE) effects in motor experts as compared with novices. $\mathrm{L}=$ left, $\mathrm{R}=$ right

recruited for performing the trained movements. In the current meta-analysis, motor experts and novices indicated different
ALE effects in the bilateral cerebellum, and this might indicate that the two groups require different efforts for motor task
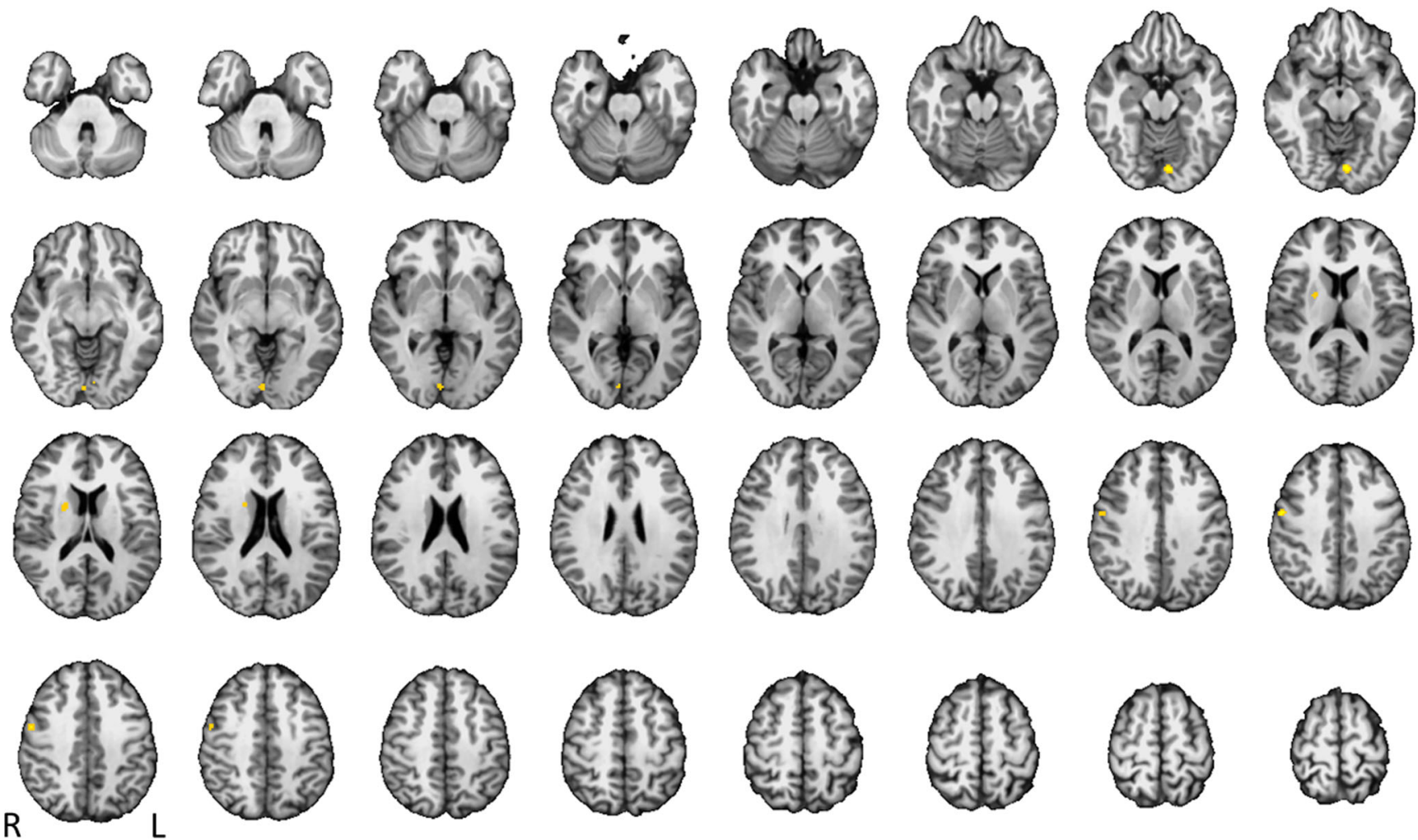

Fig. 4 Stronger activation likelihood estimation (ALE) effects in novices as compared with motor experts. $\mathrm{L}=$ left, $\mathrm{R}=$ right 
Table 5 Meta-analysis results of the motor expertise effects in different task conditions

\begin{tabular}{|c|c|c|c|c|c|c|}
\hline Volume & $\mathrm{x}$ & $\mathrm{y}$ & $\mathrm{z}$ & Hemisphere & Region & BA \\
\hline \multicolumn{7}{|c|}{ Motor execution } \\
\hline \multicolumn{7}{|c|}{ Motor experts $>$ novices (4 studies, 32 foci) } \\
\hline 328 & -42 & -32 & 46 & Left & Inferior Parietal Lobule & 40 \\
\hline \multicolumn{7}{|c|}{ Novices $>$ motor experts ( 2 studies, 47 foci) } \\
\hline 352 & -4 & -80 & -12 & Left & Cerebellum & \\
\hline 248 & 24 & -2 & 14 & Right & Putamen & \\
\hline \multicolumn{7}{|c|}{ Motor observation } \\
\hline \multicolumn{7}{|c|}{ Motor experts $>$ novices ( 4 studies, 140 foci) } \\
\hline 320 & -42 & -2 & 28 & Left & Precentral Gyrus & 6 \\
\hline 256 & -56 & 8 & 30 & Left & Inferior Frontal Gyrus & 9 \\
\hline \multicolumn{7}{|c|}{ Novices $>$ motor experts (N/A) } \\
\hline \multicolumn{7}{|c|}{ Motor prediction } \\
\hline \multicolumn{7}{|c|}{ Motor experts > novices ( 3 studies, 50 foci) } \\
\hline 480 & 6 & -74 & -28 & Right & Cerebellum & \\
\hline 228 & -34 & -48 & 38 & Left & Inferior Parietal Lobule & 40 \\
\hline \multicolumn{7}{|c|}{ Novices $>$ motor experts ( 3 studies, 21 foci) } \\
\hline 204 & 0 & -56 & 56 & Left & Precuneus & 7 \\
\hline
\end{tabular}

performance. Whether the effect difference in the cerebellum indicates that motor experts and novices have different efficiency during task performance needs further investigation.

Although the current meta-analysis indicates that motor experts show cortical reorganization during motor task performance as compared with novices, several factors might influence the results. These factors need to be investigated in future studies. First, different types of motor training might induce different cortical reorganization. For instance, music-related motor training (e.g., playing a piano, playing a keyboard, and drumming) usually requires auditory feedback. Thus, the coupling between auditory input and motor output increases during motor training, and the functional connectivity between the auditory cortex and motor cortex might increase accordingly. It has been found that for musicians with strong motor skills, cortical reorganization occurs not only in motor areas, but also in auditory areas (Baumann et al., 2007). For instance, Baumann et al. (2007) studied audio-motor coordination networks in pianists and non-musicians during a silent piano performance and while listening to piano sound. The authors found that both groups showed concurrent activation in the secondary auditory cortex (e.g., planum temporale), secondary motor cortex (e.g., premotor cortex and SMA), and high-order motor planning regions (e.g., IPL). Furthermore, pianists showed stronger activity in the right dorsal premotor cortex and left pre-SMA. This finding suggests that intensive music training can strengthen the interaction between auditory and motor areas. On the other hand, auditory feedback is not necessary for many sport-related motor training, but visual and somatosensory feedback are quite important. Thus, sport-related motor expertise might induce cortical reorganization in motor, visual, and somatosensory areas. The current meta-analysis is unable to test these hypotheses due to the limited number of studies. However, future research should examine how different types of motor training can influence the cortical reorganization underpinning motor task performance.

Another factor that might have influenced the current results comes is the exclusion of ROI findings in previous research. In the current study, only activations from wholebrain analysis were included, and findings from ROI analysis were excluded. In ALE meta-analysis, all input coordinates are put into a whole-brain mask to calculate the ALE map for a certain contrast. Subsequently, the computed ALE map is compared with a whole-brain null distribution to distinguish significant convergence from noise (Eickhoff et al., 2009). Because ALE is conducted within a whole-brain mask, it is necessary to select coordinates from whole-brain analysis in individual studies. However, a potential disadvantage is that findings from ROI analysis cannot be included. These ROI findings provide important information for the training-related functional reorganization. For example, Koeneke et al. (2004) found significant differences between pianists and nonmusicians in the whole-brain analysis. Nevertheless, when the analysis was restricted to the a priori defined cerebellar volume, the authors found significant differences between pianists and non-musicians within the vermis and in the cerebellar cortices. This cerebellar effect could not be included in the current meta-analysis because the statistical analysis was done in the cerebellar mask, not in the whole-brain mask. 
The fact that ROI findings cannot be included in the ALE meta-analysis might be related to the inconsistency between the current result and some previous findings showing that motor experts have reduced brain effects in training-related areas. Although some ROI findings indicate that motor training induces stronger activity in task-related areas (e.g., Calvo-Merino et al., 2005), other ROI effects show that motor training can induce activation decreases. For example, Krings et al. (2000) found that piano players had less significant voxels in four ROIs (i.e., primary sensorimotor cortex, SMA, superior parietal area, and premotor area) as compared with novices. Milton et al. (2007) conducted ROI analysis in anatomical regions and found that expert golfers had reduced activation volume in widespread brain regions as compared with novices, including SMA, lateral premotor cortex, M1, and MFG. These ROI results (i.e., activation volume in experts and novices) indicate that motor experts have less activation than novices in taskrelated areas, but they cannot be included in ALE metaanalysis that is based on coordinate results from wholebrain analysis.

Finally, although the fMRI technique has several advantages (e.g., good spatial resolution, whole-brain data acquisition, and recording cortical and subcortical activity similarly) and has been widely used in research about training-related plasticity, it has shortcomings and needs to be complemented by neurophysiological measures (e.g., EEG and TMS). The main measure of interest in fMRI is blood oxygenation leveldependent (BOLD) response. This causes two disadvantages: low temporal resolution and indirect measures of neural activity. Blood flow is slow relative to neural activity, and the BOLD signal is susceptible to influences of non-neural changes in the body. Thus, it is hard to use fMRI to measure training-related plasticity within a short time frame, and the training-related BOLD effect may reflect influences of other effects rather than training effects. In addition, different fMRI studies use different field strength, voxel size, and field of view. This may make the data heterogeneous, and thus it is hard to obtain really comparable spatial resolution for the meta-analysis.

Neurophysiological techniques can complement the fMRI technique when studying training-related plasticity (Herholz \& Zatorre, 2012). EEG measures evoked sensory responses and the synchronization of neural activity. TMS causes depolarization or hyperpolarization in the neurons of the brain. Both techniques can directly measure or modify neural activity, and they have good temporal resolution. In addition, the EEG technique allows whole-brain data acquisition and network analysis, but its spatial resolution is low. TMS has high spatial resolution, but can only work on local regions. A disadvantage of both techniques is that they are less sensitive to deeper and subcortical structures than to surface and cortical structures.
It is clear that no single technique can fully characterize training-induced plasticity in the brain. Only the combination of fMRI and neurophysiological techniques can measure brain plasticity temporally and spatially, at both cortical and subcortical levels.

\section{Conclusion}

The current study shows that motor expertise increases brain effects in areas related to action planning and action comprehension and decrease brain effects in right brain areas and areas related to motor control. These results suggest that intensive motor training strengthens the involvement of taskrelated areas, probably because motor training develops a more elaborate motor representation related to the tasks. However, how motor expertise changes the attention process during motor task performance still needs investigation. Future research may also focus on the influences of other factors (e.g., different sensory feedbacks) on the functional reorganization induced by motor training experience.

\section{References}

Abreu, A. M., Macaluso, E., Azevedo, R. T., Cesari, P., Urgesi, C., \& Aglioti, S. M. (2012). Action anticipation beyond the action observation network: A functional magnetic resonance imaging study in expert basketball players. European Journal of Neuroscience, 35, 1646-1654.

Allard, F., \& Starkes, J. L. (1991). Motor-skill experts in sports, dance, and other domains. In K. A. Ericsson \& J. Smith (Eds.), Toward a general theory of expertise: Prospects and limits (pp. 126-152). Cambridge: Cambridge University Press.

Balser, N., Lorey, B., Pilgramm, S., Stark, R., Bischoff, M., Zentgraf, K., ..., \& Munzert, J. (2014). Prediction of human actions: Expertise and task-related effects on neural activation of the action observation network. Human Brain Mapping, doi: 10.1002/hbm.22455

Baeck, J. S., Kim, Y. T., Seo, J. H., Ryeom, H. K., Lee, J., Choi, S. M., ..., \& Chang, Y. (2012) Brain activation patterns of motor imagery reflect plastic changes associated with intensive shooting training. Behavioral Brain Research, 234, 26-32.

Baumann, S., Koeneke, S., Schmidt, C. F., Meyer, M., Lutz, K., \& Jancke, L. (2007). A network for audio-motor coordination in skilled pianists and non-musicians. Brain Research, 1161, 65-78.

Beilock, S. L., Lyons, I. M., Mattarella-Micke, A., Nusbaum, H. C., \& Small, S. L. (2008). Sports experience changes the neural processing of action language. Proceedings of the National Academy of Sciences of the United States, 105, 13269-13273.

Berkowitz, A. L., \& Ansari, D. (2010). Expertise-related deactivation of the right temporoparietal junction during musical improvisation. NeuroImage, 49, 712-719.

Bernardi, G., Ricciardi, E., Sani, L., Gaglianese, A., Papasogli, A., Ceccarelli, R., ... \& P Pietrini, P. (2013). How skill expertise shapes the brain functional architecture: an fMRI study of visuo-spatial and motor processing in professional racing-car and naïve drivers. $P L O S$ One, 8, e77764. 
Bezzola, L., Merillat, S., \& Jäncke, L. (2012). Motor training-induced neuroplasticity. The Journal of Gerontopsychology and Geriatric Psychiatry, 25, 189-197.

Binkofski, F., Buccino, G., Posse, S., Seitz, R. J., Rizzolatti, G., \& Freund, H. J. (1999). Afronto-parietal circuit for object manipulation in man: Evidence from an fMRI-study. European Journal of Neuroscience, 11, 3276-3286.

Bishop, D. T., Wright, M. J., Jackson, R. C., \& Abernethy, B. (2013). Neural bases for anticipation skill in soccer: An FMRI study. Journal of Sport \& Exercise Psychology, 35, 98-109.

Calvo-Merino, B., Glaser, D. E., Grèzes, J., Passingham, R. E., \& Haggard, P. (2005). Action observation and acquired motor skills: An fMRI study with expert dancers. Cerebral Cortex, 15, 1243-1249.

Chang, Y., Lee, J.J., Seo, J. H., Song, H. J., Kim, Y. T., Lee, H. J., ..., \& Kim, J. G. (2011). Neural correlates of motor imagery for elite archers. NMR in Biomedicine, 24, 366-372.

Chouinard, P. A., \& Paus, T. (2006). The primary motor and premotor areas of the human cerebral cortex. The Neuroscientist, 12, 143-152.

Decety, J., Perani, D., Jeannerod, M., Bettinardi, V., Tadary, B., Woods, R., Mazziotta, J. C., \& Fazio, F. (1994). Mapping motor representations with positron emission tomography. Nature, 371, 600-602.

Eickhoff, S. B., Laird, A. R., Grefkes, C., Wang, L. E., Zilles, K., \& Fox, P. T. (2009). Coordinate-based activation likelihood estimation meta-analysis of neuroimaging data: A random-effects approach based on empirical estimates of spatial uncertainty. Human Brain Mapping, 30, 2907-2926.

Garavan, H., Kelley, D., Rosen, A., Rao, S. M., \& Stein, E. A. (2000). Practice-related functional activation changes in a working memory task. Microscopy Research \& Technique, 51, 54-63.

Grafton, S. T., Arbib, M. A., Fadiga, L., \& Rizzolatti, G. (1996). Localization of grasp representations in humans by positron emission tomography. 2. Observation compared with imagination. Experimental Brain Research, 112, 103-111.

Grezes, J., \& Decety, J. (2002). Does visual perception of object afford action? Evidence from a neuroimaging study. Neuropsychologia, 40, 212-222.

Harris, R., \& de Jong, B. M. (2014). Cerebral activations related to audition-driven performance imagery in professional musicians. PLoS One, 9, e93681.

Hasegawa, T., Matsuki, K., Ueno, T., Maeda, Y., Matsue, Y., Konishi, Y., \& Sadato, N. (2004). Learned audiovisual cross-modal associations in observed piano playing activate the left planum temporale. An fMRI study. Brain Research Cognitive Brain Research, 20, 510-8.

Haslinger, B., Erhard, P., Altenmüller, E., Hennenlotter, A., Schwaiger, M., Gräfin von Einsiedel, ..., \& Ceballos-Baumann, A. O. (2004). Reduced recruitment of motor association areas during bimanual coordination in concert pianists. Human Brain Mapping, 22, 206215 .

Haslinger, B., Erhard, P., Altenmüller, E., Schroeder, U., Boecker, H., \& Ceballos-Baumann, A. O. (2005). Transmodal sensorimotor networks during action observation in professional pianists. Journal of Cognitive Neuroscience, 17, 282-293.

Herholz, S. C., \& Zatorre, R. J. (2012). Musical training as a framework for brain plasticity: Behavior, function, and structure. Neuron, 76, 486-502.

Hoshi, E., \& Tanji, J. (2007). Distinctions between dorsal and ventral premotor areas: Anatomical connectivity and functional properties. Current Opinion of Neurobiology, 17, 234-242.

Jäncke, L. (2009a). Music drives brain plasticity. F1000 Biology Reports, $1,78$.

Jäncke, L. (2009b). The plastic human brain. Restorative Neurology and Neuroscience, 27, 521-538.

Johnson, P. B., Ferraina, S., Bianchi, L., \& Caminiti, R. (1996). Cortical networks for visual reaching: Physiological and anatomical organization of frontal and parietal lobe arm regions. Cerebral Cortex, 6, 102-119.

Jonides, J. (2004). How does practice makes perfect? Nature Neuroscience, 7, 10-11.
Kelly, A. M., \& Garavan, H. (2005). Human functional neuroimaging of brain changes associated with practice. Cerebral Cortex, 15, 1089 1102 .

Kim, J., Lee, H. M., Kim, W. J., Park, H. J., Kim, S. W., Moon, D. H., ..., \& Tennant, L. K. (2008). Neural correlates of pre-performance routines in expert and novice archers. Neuroscience Letters, 445, 236-241.

Kim, Y. T., Seo, J. H., Song, H. J., Yoo, D. S., Lee, H. J., Lee, J., ..., \& Chang, Y. (2011). Neural correlates related to action observation in expert archers. Behavioral Brain Research, 223, 342-347.

Koeneke, S., Lutz, K., Wüstenberg, T., \& Jäncke, L. (2004). Long-term training affects cerebellar processing in skilled keyboard players. Neuroreport, 15, 1279-1282.

Konen, C. S., Mruczek, R. E. B., Montoya, J. L., \& Kastner, S. (2013). Functional organization of human posterior parietal cortex: Grasping-and reaching-related activations relative to topographically organized cortex. Journal of Neurophysiology, 109, 2897-2908.

Krings, T., Töpper, R., Foltys, H., Erberich, S., Sparing, R., Willmes, K., \& Thron, A. (2000). Cortical activation patterns during complex motor tasks in piano players and control subjects. A functional magnetic resonance imaging study. Neuroscience Letters, 278, 189-193.

Lancaster, J. L., Tordesillas-Gutiérrez, D., Martinez, M., Salinas, F., Evans, A., Zilles, K., ..., \& Fox, P. T. (2007). Bias between MNI and Talairach coordinates analyzed using the ICBM-152 brain template. Human Brain Mapping, 28, 1194-1205.

Landau, S. M., \& D'esposito, M. (2006). Sequence learning in pianists and nonpianists: An fMRI study of motor expertise. Cognitive, Affective, and Behavioral Neuroscience, 6, 246-259.

Lee, H., \& Noppeney, U. (2011). Long-term music training tunes how the brain temporally binds signals from multiple senses. Proceedings of the National Academy of Sciences of the United States, 108, E1441-1450.

Llinás, R. R. (2011). Cerebellar motor learning versus cerebellar motor timing: The climbing fibre story. Journal of Physiology, 589, 34233432

Lyons, I. M., Mattarella-Micke, A., Cieslak, M., Nusbaum, H. C., Small, S. L., \& Beilock, S. L. (2010). The role of personal experience in the neural processing of action-related language. Brain and Language, 112, 214-222.

Meister, I., Krings, T., Foltys, H., Boroojerdi, B., Müller, M., ..., \& Thron, A. (2005). Effects of long-term practice and task complexity in musicians and nonmusicians performing simple and complex motor tasks: implications for cortical motor organization. Human Brain Mapping, 25, 345-352.

Milton, J., Solodkin, A., Hlustík, P., \& Small, S. L. (2007). The mind of expert motor performance is cool and focused. NeuroImage, 35, 804-813.

Nakata, H., Yoshie, M., Miura, A., \& Kudo, K. (2010). Characteristics of the athletes' brain: Evidence from neurophysiology and neuroimaging. Brain Research Review, 62, 197-211.

Newman-Norlund, R., van Schie, H. T., van Zuijlen, A. M. J., \& Bekkering, H. (2007). The mirror neuron system is more active during complementary compared with imitative action. Nature Neuroscience, 10, 817-818.

Olsson, C. J., \& Lundström, P. (2013). Using action observation to study superior motor performance: A pilot fMRI study. Frontiers in Human Neuroscience, 7, 819.

Pau, S., Jahn, G., Sakreida, K., Domin, M., \& Lotze, M. (2013). Encoding and recall of finger sequences in experienced pianists compared with musically naïve controls: A combined behavioral and functional imaging study. NeuroImage, 64, 379-87.

Penny, W. D., \& Holmes, A. P. (2004). Random effects analysis. In R. S. J. Frackowiak, K. J. Friston, R. Frith, K. J. Dolan, C. J. Price, S. Zeki, J. Ashburner, \& W. D. Penny (Eds.), Human brain function (pp. 843-850). San Diego: Academic.

Petrini, K., Pollick, F. E., Dahl, S., McAleer, P., McKay, L. S., Rocchesso, D., ..., Puce, A. (2011). Action expertise reduces brain activity for 
audiovisual matching actions: an fMRI study with expert drummers. Neuroimage, 56, 1480-1492.

Pilgramm, S., Lorey, B., Stark, R., Munzert, J., Vaitl, D., \& Zentgraf, K. (2010). Differential activation of the lateral premotor cortex during action observation. BMC Neuroscience, 11, 89.

Rizzolatti, G., Cattaneo, L., Fabbri-Destro, M., \& Rozzi, S. (2014). Cortical mechanisms underlying the organization of goal-directed actions and mirror neuron-based action understanding. Physiological Reviews, 94, 655-706.

Seo, J., Kim, Y. T., Song, H. J., Lee, H. J., Lee, J., Jung, T. D., ..., \& Chang, Y. (2012). Stronger activation and deactivation in archery experts for differential cognitive strategy in visuospatial working memory processing. Behavioral Brain Research, 229, 185-93.

Stoodley, C. J. (2012). The cerebellum and cognition: Evidence from functional imaging studies. Cerebellum, 11, 352-365.

Stout, D., Passingham, R., Frith, C., Apel, J., \& Chaminade, T. (2011). Technology, expertise and social cognition in human evolution. European Journal of Neuroscience, 33, 1328-1338.

Talairach, J., \& Tournoux, P. (1988). Co-planar stereotaxic atlas of the human brain. New York: Thieme Medical.

Tomasino, B., Maieron, M., Guatto, E., Fabbro, F., \& Rumiati, R. I. (2013). How are the motor system activity and functional connectivity between the cognitive and sensorimotor systems modulated by athletic expertise? Brain Research, 1540, 21-41.

Vingerhoets, G. (2014). Contribution of the posterior parietal cortex in reaching, grasping, and using objects and tools. Frontiers in Psychology, 5, 151.

Wei, G., \& Luo, J. (2010). Sport expert's motor imagery: Functional imaging of professional motor skills and simple motor skills. Brain Research, 1341, 52-62.

Wright, M. J., Bishop, D. T., Jackson, R. C., \& Abernethy, B. (2013). Brain regions concerned with the identification of deceptive soccer moves by higher-skilled and lower-skilled players. Frontiers in Human Neuroscience, 7, 851.

Wright, M. J., Bishop, D. T., Jackson, R. C., \& Abernethy, B. (2011). Cortical fMRI activation to opponents' body kinematics in sportrelated anticipation: Expert-novice differences with normal and point-light video. Neuroscience Letters, 500, 216-221.

Wright, M. J., Bishop, D. T., Jackson, R. C., \& Abernethy, B. (2010). Functional MRI reveals expert-novice differences during sportrelated anticipation. Neuroreport, 21, 94-98.

Zatorre, R. J., Fields, R. D., \& Johansen-Berg, H. (2012). Plasticity in gray and white: Neuroimaging changes in brain structure during learning. Nature Neuroscience, 15, 528-536. 